\title{
‘Oh, Baby, Please Don't Cry!': In Infants Suffering from Infantile Colic Hypothalamic-Pituitary- Adrenocortical Axis Activity Is Related to Poor Sleep and Increased Crying Intensity
}

\author{
Serge Brand ${ }^{a}$ Raoul Furlano ${ }^{b}$ Marc Sidler ${ }^{b}$ Juergen Schulz ${ }^{c}$ \\ Edith Holsboer-Trachsler ${ }^{a}$ \\ ${ }^{a}$ Depression Research Unit, Psychiatric Hospital of the University of Basel, b University Children's Hospital Basel, and \\ 'Institute of Osteopathy, Basel, Switzerland
}

\section{Key Words}

Infantile colic · Salivary cortisol · Crying behavior - Sleep •

Actigraphy $\cdot$ Hypothalamic-pituitary-adrenocortical axis

\begin{abstract}
Background/Aim: Infantile colic (IC) is considered to represent the upper end of the spectrum of early developmental crying behavior. Little is known about hypothalamic-pituitary-adrenocortical axis activity and sleep in relation to infants' crying. The aim of the present study was to assess cortisol secretion in infants in relation to their sleep and crying patterns. Method: Sixteen infants (mean age: 8 weeks; SD = 1.5 weeks) were enrolled. Their mothers completed a series of questionnaires regarding the infants' crying and sleeping patterns. The infants' sleep was objectively assessed with actigraphs. After 4 weeks, the infants were assessed once again. Cortisol secretion was measured by means of saliva samples in the mornings after awakening. Results: Morning saliva cortisol levels were related to more frequent awakening and to increased crying intensity, but not to sleep or crying duration. Over 4 weeks, both crying behavior and sleep duration decreased, but there was no association between them. Cortisol secretion did not significantly change. Conclusions: In infants suffering from IC, fragmented sleep pat-
\end{abstract}

terns and increased saliva cortisol levels were related. Cortisol secretion seems to be related to crying intensity, but not to crying duration. Crying intensity may reflect greater physiological or psychological stress rather than mere duration of crying.

Copyright $\odot 2011$ S. Karger AG, Basel

\section{Introduction}

Infantile colic (IC), colic syndrome [1], or unsoothable crying [2] is considered to represent the upper end of the range of early developmental crying behavior. IC seems to be independent of parenting behavior [3] and culture [4]. That is to say, IC is observed irrespective of either parents' behavior or the sociocultural context of childrearing. Following Wessel's seminal definition, an infant suffers from IC (colic syndrome/unsoothable crying) if crying occurs for 3 or more hours per day, for 3 or more days per week, and for 3 consecutive weeks [5]. Within the first 12 weeks of life, infants normally cry for $1-2 \mathrm{~h}$ per day, with a peak of about $3 \mathrm{~h}$ at the age of $6-8$ weeks

Serge Brand and Raoul Furlano contributed equally to this paper.

\section{KARGER}

Fax +4161306 1234 E-Mail karger@karger.ch www.karger.com
(C) 2011 S. Karger AG, Base

0302-282X/11/0641-0015\$38.00/0

Accessible online at:

www.karger.com/nps
Serge Brand, $\mathrm{PhD}$

Psychiatric Hospital of the University of Basel

Depression and Sleep Research Unit

Wilhelm Klein-Strasse 27, CH-4025 Basel (Switzerland)

Tel. +41 6132551 14, Fax +41 6132555 13, E-Mail serge.brand@ upkbs.ch 
[6]. Such crying tends to return to more normal and stable levels by around 16-20 weeks [6]. Although the term IC suggests that gastrointestinal tract problems are causally linked to crying behavior, gastrointestinal and organic problems have been detected in less than $5 \%$ of infants with IC $[7,8]$. Prolonged periods of crying are regarded rather as individual differences in the maturation of the central nervous system [9].

IC causes distress both for the infant and the caregiver. Generally, caregivers seem to be concerned both that infants are suffering from terrible pain and that they are not sleeping enough. In particular, it seems justified to be concerned that insufficient sleep is associated with poor physiological and psychological functioning. Restoring sleep is an essential precondition for physical growth and health, for behavioral and emotional stability, and for cognitive function. These observations are true not only for adults [10] and adolescents [11], but above all also for preschool children [12-14] and for toddlers [14].

With regard to a regular sleep/wake pattern, circadian and ultradian processes begin to develop in the newborn period, and a circadian rhythm emerges at around 2-3 months of age [15], with a synchronization of the sleep/ wake cycle in relation to light/dark cycles and social stimuli such as feeding $[15,16]$. It is apparent that synchronization of the sleep/wake cycle also emerges with increasing IC, but there is no clear-cut pattern of sleep in infants with IC as compared to healthy controls. Whereas Kirjavainen et al. [17] observed no statistical difference in objectively assessed sleep duration or fragmentation between infants with and without IC over a period of $24 \mathrm{~h}$, others have reported decreased sleep during the day $[18$, 19]. These conflicting results may reflect methodological differences, insofar as both subjective (i.e. sleep logs) and objective (i.e. actigraphs) assessment tools were employed. To limit the possibility of subjective bias, we took this issue into account and assessed the infants' sleep by means of actigraphy.

Another factor to bear in mind is that the interval between 8 and 16 weeks of life is marked by a period of tremendous behavioral reorganization in infants [20]. This reorganization is recognized to occur as a function of neuronal developments such as cell expansion [21].

Circadian rhythmicity, sleep, and endocrine processes are associated, and more robust patterns are seen at approximately 3 months of age [22]. Within the neuronal and neuroendocrine system, the activity of the hypothalamic-pituitary-adrenocortical (HPA) system demands particular attention. The most prominent outcome measure of HPA axis activity is cortisol secretion [21-23]. The
HPA axis enables the organism to cope specifically and individually with internal and external stressors (for an extensive overview, see Jansen et al. [24]). In this regard, Gruber et al. [25] demonstrated a significant neuroendocrine stress response in infants with well-compensated congenital cardiac disease undergoing cardiac surgery, but without adverse postoperative outcome. In a similar vein, Nakamura et al. [26] compared HPA axis reactivity to surgical stress of 19 neonates, 19 infants and 20 preschool children undergoing major thoracic and abdominal surgery. Regardless of age, cortisol levels reached a peak value just after surgery. These results suggest that in infants younger than 6 months, the HPA axis predominantly reacts in response to physical rather than psychological stressors [24]. However, there is also evidence that cortisol secretion patterns in infants and young children following involvement of Child Protective Service are altered by parents' care, with more unstable and elevated cortisol levels in those children living again with their birth parents, compared to children placed in foster care following intervention by child protection services [27].

Cortisol peak values seem significantly lower in neonates than in infants and preschool children [2] (see also Tollenaar et al. [23] for opposite results). Therefore, it was hypothesized that the neuroendocrine transmission system in newborns is still immature and that cortisol secretion periodicity is established postnatally. Indeed, in the first 4 postnatal weeks, cortisol secretion periodicity increases from $6.4 \mathrm{~h}$ with 2 daily peaks to $11.2 \mathrm{~h}$ with 1 daily peak [21]. By about 8 weeks of age, the HPA system appears to be sufficiently mature to produce a 24 -hour periodicity, but this periodicity is not clearly synchronized with the light/dark cycle. Synchronization and coordination with emerging circadian rhythmicity in night time sleep patterns increase from 8 weeks onwards and adult-like coordination of HPA periodicity, light/dark cycle, and night time sleep rhythms begin to emerge by $12-$ 16 weeks. With this synchronization, basal cortisol levels increasingly become the peak levels seen during the day [28]. Therefore, the conjunction of increased circadian and day/night organization at around 3 months and the change of cortisol secretion to a 24 -hour periodicity suggest that these 2 phenomena are functionally related. In this respect, Larson et al. [29] showed that infants who were reported to sleep at least $6 \mathrm{~h}$ or more continuously through the night had a more marked circadian variation in cortisol production. Accordingly, infants with an early morning peak cortisol level were significantly older, and they were also more likely to sleep for $6 \mathrm{~h}$ or more without signaling during the night. 
The tight association between sleep pattern and HPA axis activity has been repeatedly shown in adults [30] and preschool children $[12,13]$, with poor sleep being associated with increased cortisol secretion. In neuroendocrine terms the underlying rationale is based on an increased corticotropin-releasing hormone, acting as a substantive precursor of cortisol secretion within the HPA axis and this reduces both growth hormone secretion and slowwave sleep, which, at the behavioral level, leads to decreased sleep [31, 32]. However, little is known about the relation between sleep and HPA axis activity in infants suffering from IC, and to our knowledge, only 1 study, by Prudhomme et al. [33], has investigated these parameters in parallel. They assessed 20 infants suffering from IC and 20 healthy controls. IC sufferers cried twice as much as the controls, though during medical examination (i.e. a physiological challenge stimulating HPA axis activity) HPA axis activity (i.e. cortisol secretion) of the 2 groups did not show any statistical difference. Moreover, under baseline conditions, saliva cortisol levels over a period of 2 consecutive days showed no statistical differences between infants suffering from IC and healthy controls. Even though descriptively the infants suffering from IC displayed a blunted rhythm of cortisol production compared to healthy controls, the cortisol secretion of the infants suffering from IC was not associated with daily behavior, crying or sleeping patterns. Nonetheless, Prudhomme et al. [33] concluded that IC might be associated with incongruence 'in activity of the hypothalamic-pituitary-adrenocortical axis and associated sleep-wake activity' [33, p. 862].

Taken together, there is some evidence that infants suffering from IC do sleep less than controls and this is probably associated with HPA axis activity. However, nothing is known about the development of the relations between sleep, crying behavior, and HPA axis activity (i.e. cortisol secretion) over time in children suffering from IC. Therefore, the aim of the present study was to assess sleep, crying behavior and cortisol secretion over a period of 4 weeks. Findings are potentially important in identifying infants at increased risk, and may allow insight into the interplay between HPA axis activity and sleep during early development.

The following 3 research questions were formulated. First, on the basis of a substantial body of previous research on adults and preschool children [12, 13, 30], we anticipated that poor sleep would be associated with greater HPA axis activity [31, 32]. Second, we wished to replicate the observation that cortisol secretion is not related to crying behavior, as indicated by the findings of
Prudhomme et al. [33]. Third, as suggested by Brazelton [6], we expected that crying would decrease over time.

Since the study questions could be answered in assessing exclusively children suffering from IC, we did not recruit and assess a control group consisting of healthy children not suffering from IC.

\section{Methods}

\section{Study Population}

A total of 16 infants suffering from IC (10 female and 6 male infants; average age: 8 weeks, $\mathrm{SD}=1.5$ weeks) were prospectively enrolled in the study. All children were full-term; mean birth weight was 3,618 $\mathrm{g}(\mathrm{SD}=327.61)$, and mean APGAR scores were $9-10 / 8-10 / 8-10$. Five of the 16 children $(31.25 \%)$ were born by Cesarean section. The mothers' mean age was 33.04 years $(\mathrm{SD}=5.1$ years), and no mother reported any pregnancy or birth complications. Also, no mother reported suffering from maternal postnatal depression. For 9 of the 16 mothers (56.25\%), the child suffering from IC was the first child. Mothers who indicated that their infant cried continuously were referred by general practitioners and pediatricians to the 2 responsible physicians (R.F., M.S.) of the department of gastroenterology at the University Children's Hospital Basel (Switzerland).

We fully acknowledge that both mother and father are highly involved in rearing and caring for an infant. However, at least for the present sample and the present study, clinical practice showed that the mothers took the initiative in contacting the University Children's Hospital Basel, in keeping the logs, and in performing the study at home with the infants. For these reasons, we decided to stress the wording 'mother' instead of 'parents'. In this respect, recent findings showed that compared to fathers, mothers' saliva cortisol levels did correlate to a higher degree with infants' saliva cortisol levels, suggesting that mothers and infants might share similar environmental conditions [34].

The parents were fully informed about the purpose and experimental details of the study and signed an informed consent form. A thorough clinical examination of the infants was performed to exclude somatic and pathological signs as possible reasons for crying. Nineteen children were examined and 3 were excluded due to somatic illnesses such as acute airway infection and overt gastroesophageal reflux disease. The study was conducted in accordance with the Declaration of Helsinki, and the experimental protocol was approved by the local ethics committee of Basel.

\section{Study Procedure}

After initial diagnosis of IC, the infants' crying and sleep were assessed (see detailed descriptions below) for 5 consecutive days and nights as the first time point. To assess HPA axis activity, on the last morning of the fifth day 4 saliva samples were obtained to analyze the cortisol level. Next, experts (R.F., M.S.) gave advice to the mothers on how to cope with the infant's crying and sleeping behavior, including advice and counseling regarding calming down the infant, seeking aid from partners, parents, neighbors, or friends in case of desperation and great distress, or gently lying the crying infant on the floor and leaving the room instead of running the risk of shaking the infant. Moreover, the mothers were 
free to contact R.F. and M.S. in case of any further need. The counseling was not intended as training, though it would not have been ethical not to give any support. Four weeks later, the infants' crying and sleep were assessed once again for 5 consecutive days and nights. Again, HPA axis activity was assessed on the last morning of the last day, that is, again, 4 saliva samples were obtained to analyze the cortisol level (second time point).

\section{Assessment of IC/Crying Behavior}

An infant was considered to suffer from IC, if she/he fulfilled Wessel's 'Rule of Three' [5], that is: crying for about $3 \mathrm{~h}$ for at least 3 consecutive days within 3 or more consecutive weeks, and provided that any somatic illness was completely excluded. At the first telephone contact, the mothers of infants suffering from possible IC were asked to record crying periods in a log until their first visit, in order to quantify the infants' crying. Moreover, they had to report that the infant was quick to cry and difficult to soothe. After the first consultation, the mothers were asked and trained to keep a further log consisting of: (a) crying duration: every 24-hour period was graphically divided into a grid of 15 -min boxes. The mothers ticked the boxes whenever the infant was crying; (b) crying volume: answers were given on a 9-point rating scale with the anchor points 1 (= very low, practically inaudible) to 9 (= very loud); (c) crying intensity: answers were given on a 9-point rating scale with the anchor points 1 (= very soft whining; inconspicuous facial expression) to 9 (= very intense crying; infant grimacing in pain). The crying log was kept for 5 consecutive days and nights. A composite score was calculated separately, with higher scores indicating longer, louder and more intense crying.

\section{Sleep Assessment}

Sleep was assessed in 2 ways. First, the mothers were trained to keep a daily log of their infant's sleep. They indicated for each 30 -min period of a day whether the infant was sleeping. Sleep was defined as follows: the child's eyes are closed; breathing is regular and rhythmical; body, head, and limbs are moved only accidentally. The mothers additionally noted whether any change in sleep routine occurred. The information derived from the daily sleep log was also used to check possible discrepancies or missing data from the actigraph $[13,35]$. The sleep log was also kept for 5 consecutive days and nights.

Second, sleep was objectively assessed under in-home conditions for 5 consecutive days and nights. According to Sadeh et al. [36], in-home sleep assessment in children has the advantage that sleep does not seem to be negatively affected. The infants wore a digital movement-measuring instrument (actigraph; Somnowatch ${ }^{\circledR}$; Somnomedics, Randersacker, Germany) on the left ankle, as proposed by Acebo et al. [37]. The commercially available tool has the dimension of a wristwatch, and registers every movement above $0.012 \mathrm{~g}$ in a biaxial direction. The data, recorded in 30 -second intervals, were digitally integrated and translated into sleep measures using the software program (based on sleep/wake algorithm as defined by Gorny et al. [38], and provided by Somnowatch, Somnomedics, Germany). The mothers were fully instructed on the use of the device. They were trained to push the 'marker button' to indicate the beginning and the end of every sleeping period; that is to say, they pushed the 'marker button' whenever the infant fell asleep and whenever the infant woke up. This instruction was important because an actigraph computes sleep/wake patterns as a function of the wearer's bodily activity.
The actigraph identifies sleep when after a specific algorithm no activity is registered any more. To illustrate: the actigraph identifies erroneously the state of a calm but awake infant as sleep. Correspondingly, the actigraph registers activity and identifies the state of a sleeping infant as awake when the infant is in fact sleeping in a baby carriage or in a baby seat while being driven in a car. To avoid false hits, mothers were carefully trained to use the actigraph. If unsure whether they had pushed the button firmly enough, the mothers were instructed to push the button a second time. To become accustomed to the actigraph, the infants started wearing the instrument during an afternoon. This avoided possible first-night effects although first-night effects with actigraphs have not so far been reported [36]. Actigraphic measurements have been repeatedly shown to provide reliable data [13, 37, 39-41].

The following sleep continuity parameters were gathered [13, $35,39]$ : total sleep time during the day and at night; number of awakenings during the day and at night. Interclass correlation of night-to-night and day-to-day reliability was very high $(\mathrm{r}>0.91)$ $[13,35]$. Thus, data for the first 5 days and nights (first time point) and for the second 5 days and nights (second time point) were each combined to form composite variables.

\section{HPA System Assessment}

Morning cortisol levels have been shown to be a reliable biological marker for the HPA activity of children, adolescents, and adults when measured repeatedly with strict reference to the time of awakening [22, 42]. Therefore, 4 saliva cortisol samples were collected by the mother after the last night of actigraphy in the morning at $0,10,20$, and $30 \mathrm{~min}$ after the infant's awakening and stored in the refrigerator. Waking times ranged from 8.00 to 9.00 a.m. Feeding occurred only after saliva collection. The mothers were instructed and trained in saliva sampling before starting the study. Specifically, the mothers were trained to keep their infants in the lap in an upright position. Every cotton swab was provided with a tear-resistant nylon yarn. The mother wrapped the end of the tearresistant nylon yarn around one of her fingers and the cotton swab was placed in the infant's mouth for about $1 \mathrm{~min}$. This procedure ensured that no cotton swab could be swallowed in error.

Saliva Cortisol Sampling Technique and Cortisol Analysis

Saliva samples were obtained as described above. They were then returned to the laboratory, where samples were centrifuged at $4^{\circ} \mathrm{C}(2,000 \mathrm{rpm}, 10 \mathrm{~min})$ and stored at $-20^{\circ} \mathrm{C}$ until assay.

Free salivary cortisol concentrations were analyzed using a time-resolved immunoassay with fluorometric detection 'CoatA-Count' Cortisol RIA from DPC (Diagnostics Products Corporation; obtained through $\mathrm{H}$. Biermann $\mathrm{GmbH}$, Bad Nauheim, Germany) as described in detail elsewhere [43]. Intra- and interassay variabilities of this assay were less than 4.05 and $4.67 \%$, respectively.

Of the cortisol values sampled at 0,10,20 and $30 \mathrm{~min}$ after awakening, to compute a reliable estimate of the total amount of hormone release, the area under the concentration-time curve (AUC, nmol/l) of the morning cortisol levels was calculated using trapezoidal integration $[44,45]$. The AUC total refers to the entire amount of cortisol concentration under the time curve, whereas the AUC basal describes the initial and averaged amount of cortisol secretion over time, as if the HPA axis would not have been stimulated; accordingly, the AUC net refers to the difference in cortisol secretion between AUC total and basal $[45,46]$. 
Table 1. Descriptive and inferential overview of cortisol values, sleep patterns and crying behavior over time

\begin{tabular}{|c|c|c|c|c|c|c|}
\hline & \multicolumn{2}{|l|}{ Time points ${ }^{1}$} & \multicolumn{4}{|c|}{ Statistical comparison: $t$ tests for related samples } \\
\hline & first time point & second time point & $\mathrm{t}$ & d.f. & $\mathrm{p}$ & $d$ \\
\hline \multicolumn{7}{|l|}{ Cortisol } \\
\hline AUC total, nmol/l & $554.33(526.61)$ & $489.95(206.26)$ & 0.49 & 15 & 0.632 & 0.16 \\
\hline AUC basal, nmol/l & $368.52(591.79)$ & $311.55(178.45)$ & 0.37 & 15 & 0.729 & 0.13 \\
\hline AUC net, nmol/l & $185.82(320.97)$ & $178.40(168.56)$ & 0.08 & 15 & 0.935 & 0.02 \\
\hline \multicolumn{7}{|l|}{ Crying } \\
\hline Duration, $\mathrm{h}$ & $3.74(0.63)$ & $1.96(0.76)$ & 4.77 & 15 & 0.001 & 2.55 \\
\hline Intensity, arbitrary units & $5.43(1.05)$ & $4.22(1.23)$ & 3.07 & 15 & 0.008 & 1.05 \\
\hline Volume, arbitrary units & $5.00(1.21)$ & $4.77(0.89)$ & 0.90 & 15 & 0.381 & 0.22 \\
\hline \multicolumn{7}{|l|}{ Sleep } \\
\hline \multicolumn{7}{|l|}{ Day } \\
\hline Duration, $\mathrm{h}$ & $9.79(3.09)$ & $6.70(3.21)$ & 4.06 & 15 & 0.001 & 0.98 \\
\hline Awakenings, $\mathrm{n}$ & $40.35(15.20)$ & $27.67(12.53)$ & 3.37 & 15 & 0.004 & 0.59 \\
\hline \multicolumn{7}{|l|}{ Night } \\
\hline Duration, $\mathrm{h}$ & $11.08(2.28)$ & $8.69(2.54)$ & 3.51 & 15 & 0.003 & 0.99 \\
\hline Awakenings, $\mathrm{n}$ & $8.10(6.18)$ & $10.06(7.61)$ & 0.88 & 15 & 0.391 & 0.28 \\
\hline
\end{tabular}

Crying intensity and volume: higher values reflect greater intensity and volume. ${ }^{1}$ Figures are means with SD in parentheses.

Statistical Analyses

Pearson's correlations between cortisol values, sleep and crying behavior were performed. To calculate changes over time of cortisol, sleep, and crying behavior, a series of $t$ tests for related samples was performed. Test results with an alpha error of below 0.05 are reported as significant. However, we placed more emphasis on effect sizes (d) following Cohen's advice [48, 49] that the importance of $\mathrm{p}$ values should not be overestimated. Effect sizes for $\mathrm{t}$ tests were calculated according to Cohen [47, 48], with $0.49 \geq d \geq 0.20$ indicating small (i.e. negligible practical importance), $0.79 \geq d \geq 0.50$ indicating medium (i.e. moderate practical importance), and $d \geq 0.80$ indicating large (i.e. crucial practical importance) effect sizes. All calculations were performed using SPSS $16.0{ }^{\circledR}$ for Windows.

\section{Results}

\section{Cortisol Secretion, Sleep Patterns and Crying}

Behavior over Time

Preliminary calculations showed that target variables (sleep, crying, cortisol values) did not systematically vary as a function of children's age, birth weight, APGAR score, gender, birth (Cesarean vs. normal birth) or birth order, nor as a function of mothers' age or delivery experience. Therefore, none of these potentially confounding factors were introduced as covariates.

Infantile Colic, Sleep and HPA
Table 1 gives a descriptive and inferential statistical overview of cortisol secretion, sleep patterns, and crying behavior.

Cortisol secretion did not decrease over time. Decreases in duration and intensity of crying over time were statistically significant. During the day, sleep duration and awakenings showed a statistically significant decrease over time. During the night, sleep duration showed a statistically significant decrease over time, whereas no statistically significant change in awakenings was observed over time.

\section{Correlations between Cortisol Secretion, Sleep \\ Patterns and Crying Behavior}

Crying duration at both time points was not related to cortisol secretion. Crying volume was related to cortisol secretion for the first, but not for the second time point. Crying intensity was positively correlated with cortisol secretion at both time points (fig. 1a).

For the first time point, sleep duration and number of awakenings during the day and during the night were unrelated to cortisol secretion.

For the second time point, sleep during the day (duration, awakenings) was not related to cortisol secretion. However, prolonged sleep during the night was related to increased cortisol secretion (AUC total; AUC net; fig. 1b). 

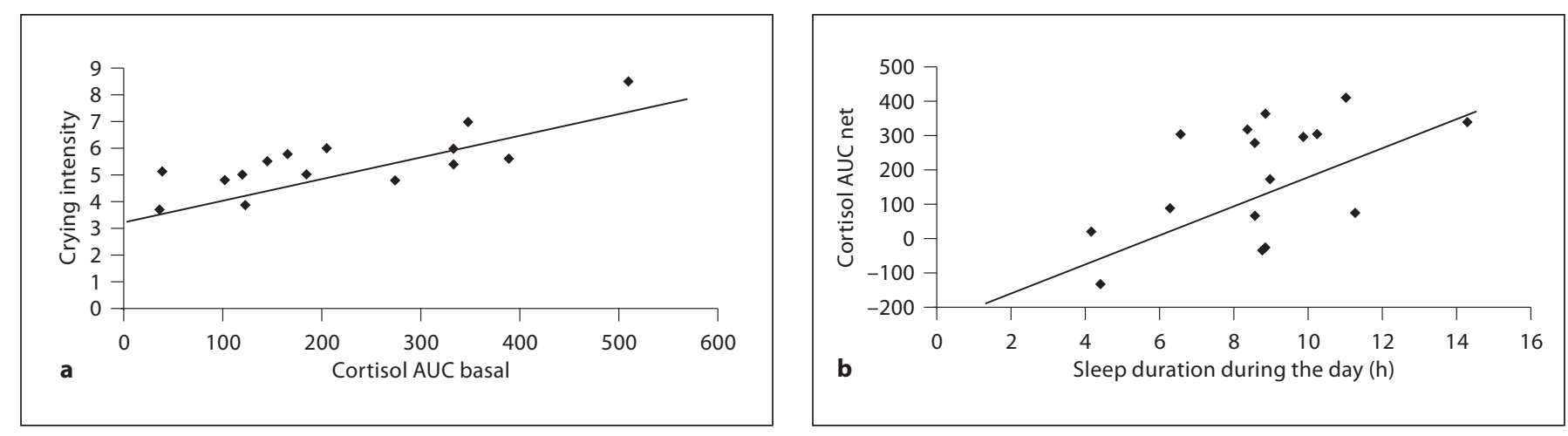

Fig. 1. a Increased cortisol AUC basal (first time point) was related to greater crying intensity (first time point; $\mathrm{r}=0.72, \mathrm{p}<0.01$ ). $\mathbf{b}$ More prolonged sleep duration (h) during the day (second time point) was related to increased cortisol (AUC net; second time point; $\mathrm{r}=0.54, \mathrm{p}<0.05$ ).

\section{Correlations between Sleep Patterns and Crying} Behavior

No statistically significant correlations were found between sleep patterns and crying behavior (all $\mathrm{r}$ values $<0.25, \mathrm{p}>0.1)$.

\section{Discussion}

The key findings of the present study are that, in infants suffering from IC, elevated cortisol secretion was related to crying intensity as well as to prolonged sleep and to a greater number of awakenings. However, although cortisol secretion did not change over the time period of 4 weeks, sleep patterns decreased and crying behavior improved.

Three research questions were formulated and each of these is now considered in turn.

Regarding the first research question we anticipated that poor sleep would be associated with increased cortisol secretion, but data did not support this expectation. The present findings do seem to be in direct conflict with findings for adults [30] and preschool children [12, 13]. However, the pattern of results appears to be much more complex. In the present study, a higher level of cortisol secretion was related both to prolonged sleep duration, which may be considered a favorable sleep pattern, but also to more frequent awakenings, which may be considered to be an unfavorable sleep pattern. So, the pattern of results appears more complex than expected. In trying to reconcile these apparently contradictory results at least 3 explanations are possible. First, insofar as sleep is more prolonged the probability of more awakenings increases, while the presence of a disturbed sleep pattern remains unchanged. Second, overall sleep duration may have increased because the infants' sleep was more fragmented. Although our data do not provide a conclusive answer, we claim that the pattern of results may fit well with the numerous studies showing a close relation between increased HPA axis activity and poor sleep [31,32] as reflected by the more fragmented and irregular sleep already apparent in preschool children $[12,13]$. Though speculative, the present data may extend this pattern of results to infants suffering from IC. Third, the association between prolonged sleep and increased cortisol secretion may reflect a more accentuated and more maturated rhythmicity of HPA axis activity in infants. As Gunnar et al. [28] and Larson et al. [29] have shown, cortisol secretion periodicity increases from $6.4 \mathrm{~h}$ with 2 daily peaks to $11.2 \mathrm{~h}$ with 1 daily peak within the first weeks of life [21]. With this development, early morning basal cortisol levels increasingly become the peak levels during the day [28]. Therefore, the conjunction of increased circadian and day/night organization at around 3 months, and a change of cortisol secretion to a 24 -hour periodicity point to the possibility that these 2 phenomena are functionally related. In this respect, Larson et al. [29] showed that infants who were reported to sleep at least $6 \mathrm{~h}$ or more continuously through the night had a more marked circadian variation in cortisol production. Accordingly, an early morning peak in cortisol was associated with prolonged sleep without signaling during the night.

The second research aim was to reassess the finding of Prudhomme et al. [33] that cortisol secretion was not related to crying behavior. Our data did confirm the pat- 
tern of results reported by Prudhomme et al. [33] insofar as crying duration was unrelated to HPA axis activity as reflected by cortisol level in the saliva. However, our data also highlight a close association between increased HPA axis activity and crying intensity, and, for the first time point, to crying volume. Note that crying intensity was defined as crying scored within the extremes of very soft whining and inconspicuous facial expression to very intense crying with the infant grimacing in pain. Thus, the present pattern of results appears to suggest that increased HPA axis activity and intense crying, probably reflecting pain, are related. Our data do seem to support the view that, in infants younger than 6 months, HPA axis activity increases in response to both physical [24] and psychological stressors [27]. One limitation might be that in rating their infants' crying intensity and facial expression, the mothers might have overestimated their infants' mimic and presumed pain, thus introducing bias into the scoring of crying intensity. However, such assumed bias, if true, does not explain the correlation between the subjectively rated and presumed biased crying behavior rating and the increased cortisol secretion. In short, we claim that crying intensity may well mirror physiological and psychological stress of infants as reflected by increased cortisol secretion, which in turn is the outcome of a stimulated HPA axis. However, further studies are needed to replicate and extend research related to crying intensity.

Regarding the third study question, our data did confirm the expectation that crying behavior would improve over time, as reported by Brazelton [6]. Both crying duration and intensity decreased over the 4-week period, whereas crying volume remained unchanged (table 1). Our data do not allow identification of the causes of this change in behavior. Moreover, since no correlation was found between crying behavior and sleep, we assume that a favorable change in crying behavior was not related to increased sleep. As reported in table 1, sleep duration decreased both during the day and during the night, and therefore our data do not support the view that decreased crying duration implies an increase in sleep duration. Rather, though speculative, favorable maturation and effects of neuronal development such as cell expansion [49] may be implicated, although this cannot be proven with the present data. With this in mind, Barr et al. [2] consider IC to represent the upper extreme of the range of early developmental crying behavior, which is adapted and adjusted over time.

Despite the new findings, a number of limitations make generalization difficult, and require that these data should be interpreted cautiously. First, the sample size is small. However, effect size calculations are independent of sample sizes and effect sizes showed a consistent and robust pattern of results. Second, data were gathered exclusively from infants suffering from IC; there was no control group consisting of children not suffering from IC. A control group would have allowed interpretation of the present findings with rather more certainty. However, we were substantially interested in the interplay between neuroendocrine activity, sleep and crying behavior and, importantly, in the change of these patterns over time. Nonetheless, a control group of healthy children should be included in future studies. We basically understand the present study as a smaller study which nonetheless allows shedding some more light in this area of research. Third, the data may potentially be biased, because only parents of children with IC who were willing and able to complete the study procedure volunteered to participate in the study. In this view, it is of note that under in-home conditions, IC is defined by the caregiver, and this judgment may be biased by caregivers' tolerance for crying. Last, it is also conceivable that underlying, but not assessed variables contributed to the present pattern of results. In this regard, parenting may have influenced the reported results.

\section{Conclusions}

In infants suffering from IC, fragmented sleep patterns and elevated cortisol secretion were related - an association previously observed only in preschool children and adults. Therefore, it is conceivable that a common pattern linking neuroendocrine activity and sleep regulation is already emerging very early in life. Moreover, neuroendocrine activity seemed to be related to crying intensity but not to crying duration, suggesting that crying intensity rather than merely crying duration may reflect increased physiological and psychological stress.

\section{Acknowledgements}

We thank Nadine Sammer for data collection and data entry. Moreover, we thank Marielle Koenig, Ellen Quercher and Vladimir Djurdjevic for analyzing actigraphic data. Next, we are grateful to the CortLab of the University of Trier (Germany) for the cortisol analyses. Last, we thank Nick Emler (Surrey, UK) for proofreading the manuscript. 


\section{References}

1 Barr RG: Colic and crying syndromes in infants. Pediatrics 1998;102:1282-1286.

-2 Barr RG, Paterson JA, MacMartin LM, Lehtonen L, Young SN: Prolonged and unsoothable crying bouts in infants with and without colic. J Dev Behav Pediatr 2005;26: 14-23.

3 James-Roberts IS, Alvarez M, Csipke E, Abramsky T, Goodwin J, Sorgenfrei E: Infant crying and sleeping in London, Copenhagen and when parents adopt a 'proximal' form of care. Pediatrics 2006;117:e1146-e1155.

$\checkmark 4$ Talachian E, Bidari A, Rezaie MH: Incidence and risk factors for infantile colic in Iranian infants. World J Gastroenterol 2008; 14 : 4662-4666.

5 Wessel MA, Cobb JC, Jackson EB, Harris GX Jr, Detwil AC: Paroxysmal fussing in infancy, sometimes called colic. Pediatrics 1954; 14:421-435

6 Brazelton TB: Crying in infancy. Pediatrics 1962;29:579-588.

7 Gormally SM, Barr RG: Of clinical pies and clinical clues: proposal for a clinical approach to complaints of early crying and colic. Good Practice Guide 1997;3:137-153.

$\checkmark 8$ Zwart P, Vellema-Goud MGA, Brand PLP: Characteristics of infants admitted to hospital for persistent colic, and comparison with healthy infants. Acta Paediatr 2007;96:401405.

-9 Barr RG: Changing our understanding of infant colic. Arch Pediatr Adolesc Med 2002; 156:1172-1174.

10 Banks S, Dinges DF: Behavioral and physiological consequences of sleep restriction. J Clin Sleep Med 2007;3:519-528.

$\checkmark 11$ Roberts ER, Roberts CR, Duong HT: Chronic insomnia and its negative consequences for health and functioning of adolescents: a 12-months prospective study. J Adolesc Health 2008;42:294-302.

-12 Hatzinger M, Brand S, Perren S, Stadelmann $\mathrm{S}$, von Wyl A, von Klitzing K, HolsboerTrachsler E: Electroencephalographic sleep profiles and hypothalamic-pituitary-adrenocortical (HPA)-activity in kindergarten children: early indication of poor sleep quality associated with increased cortisol secretion. J Psychiatr Res 2008;42:532-543.

13 Hatzinger M, Brand S, Perren S, Stadelmann S, Wyl AV, Klitzing KV, Holsboer-Trachsler E: Sleep actigraphy pattern and behavioral/ emotional difficulties in kindergarten children: association with hypothalamic-pituitary-adrenocortical (HPA) activity. J Psychiatr Res 2010;44:253-261.

-14 Martin J, Hiscock H, Hardy P, Davey B, Wake M: Adverse associations of infant and child sleep problems and parent health: an Australian population study. Pediatrics 2007;119:947-955.
15 Sheldon SH: Sleep in infants and children; in Lee-Chiong TL, Sateia MJ, Carskadon MA (eds): Sleep Medicine. Philadelphia, Hanley and Belfus, Inc, 2002, pp 99-103.

16 Davis KF, Parker KP, Montgomery GL: Sleep in infants and young children. 1. Normal sleep. J Pediatr Health Care 2004;18:65-71.

17 Kirjavainen J, Lehtonen L, Krjavainen T, Kero P: Sleep of excessively crying infants: a 24-hour ambulatory sleep polygraphy study. Pediatrics 2004;114:592-600.

18 James-Roberts IS, Conroy S, Hurry J: Links between infant crying and sleep-waking at six weeks of age. Early Hum Dev 1997;25: 143-152.

19 Papousek M, v. Hofacker N: Persistent crying and parenting: search for a butterfly in a dynamic system. Early Dev Parent 1995;4:209_ 224.

20 Potts AL, Cheeseman JF, Warman GR: Circadian rhythms and their development in children: implications for pharmacokinetics and pharmacodynamics in anesthesia. Paediatr Anaesth 2010, E-pub ahead of print.

21 Davis EP, Granger DA: Developmental differences in infant salivary alpha-amylase and cortisol responses to stress. Psychoneuroendocrinology 2009;34:795-804.

22 Hellhammer DH, Wüst S, Kudielka BM: Salivary cortisol as a biomarker in stress research. Psychoneuroendocrinology 2009;34: 163-171.

23 Tollenaar MS, Jansen J, Beijers R, RiksenWalraven JM, de Weerth C: Cortisol in the first year of life: normative values and intraindividual variability. Early Hum Dev 2010; 86:13-16.

24 Jansen J, Beijers R, Risken-Walraven M, de Weerth C: Cortisol reactivity in young infants. Psychoneuroendocrinology 2010;35 329-338.

25 Gruber EM, Laussen PC, Casta A, Zimmerman AA, Zurakowski D, Reid R, Odegard KC, Chakravorti S, Davis PJ, McGowan FX Jr, Hickey PR, Hansen DD: Stress response in infants undergoing cardiac surgery: a randomized study of fentanyl bolus, fentanyl infusion, and fentanyl-midazolam infusion. Anesth Analg 2001;92:882-890.

-26 Nakamura M, Suita S, Yamanouchi T, Masumoto K, Ogita K, Taguchi S, Uesugi T: Cortisol and cytokine responses after surgery in different age groups of pediatric patients. Pediatr Surg Int 2003;19:194-199.

27 Bernard K, Butzin-Dozier Z, Rittenhouse J, Dozier M: Cortisol production patterns in young children living with birth parents versus children placed in foster care following involvement of Child Protective Services. Arch Pediatr Adolesc Med 2010;164:438443.
28 Gunnar MR, Brodersen L, Nachmias M, Buss K, Rigatuso J: Stress reactivity and attachment security. Dev Psychobiol 1996;29: 191-204.

29 Larson MC, White BP, Cochran A, Donzella B, Gunnar M: Dampening of the cortisol response to handling at 3 months in human infants and its relation to sleep, circadian cortisol activity, and behavioral distress. Dev Psychobiol 1998;33:327-337.

>30 Hatzinger M, Hemmeter UM, Brand S, Ising M, Holsboer-Trachsler E: Electroencephalographic sleep profiles in treatment course and long-term outcome of major depression: association with DEX/CRH-test response. J Psychiatr Res 2004;38:453-465.

31 Buckley TM, Schatzberg AF: On the interactions of the hypothalamic-pituitary-adrenal (HPA) axis and sleep: normal HPA axis activity and circadian rhythm, exemplary sleep disorders. J Clin Endocrinol Metab 2005;90: 3106-3114.

$\checkmark 32$ Steiger A: Neurochemical regulation of sleep. J Psychiatr Res 2007;41:537-552.

33 Prudhomme BP, Gunnar MR, Larson MC, Donzella B, Barr RG: Behavioral and physiological responsivity, sleep, and patterns of daily cortisol production in infants with and without colic. Child Dev 2000;71:862-877.

34 Stenius F, Theorell T, Lilja G, Scheynius A, Alm J, Lindblad F: Comparisons between salivary cortisol levels in six-months-olds and their parents. Psychoneuroendocrinology 2008;33:352-359.

35 El-Sheikh M, Buckhalt JA, Mark Cummings E, Keller P: Sleep disruptions and emotional insecurity are pathways of risk for children. J Child Psychol Psychiatry 2007;48:88-96.

36 Sadeh A, Lavie P, Scher A, Tirosh E, Epstein $\mathrm{R}$ : Actigraphic home-monitoring sleep-disturbed and control infants and young children: a new method for pediatric assessment of sleep-wake patterns. Pediatrics 1991;87: 494-799.

37 Acebo C, Sadeh A, Seifer R, Tzischinsky O, Hafer A, Carskadon MA: Sleep/wake patterns derived from activity monitoring and maternal report for healthy 1 - to 5-year-old children. Sleep 2005;28:1568-1577.

38 Gorny SW, Allen RP, Krausmann DT, Cammarata J, Earley CJ: A parametric and sleep hysteresis approach to assessing sleep and wake from wrist activity meter with enhanced frequency range. Paper presented at the 11th Annu Meet Assoc Prof Sleep Soc, San Francisco, June 10-15, 1997.

39 Sadeh A, Raviv A, Gruber R: Sleep patterns and sleep disruption in school-age children. Dev Psychol 2000;36:291-301. 
-40 Werner H, Molinari L, Guyer C, Jenni OG: Agreement rates between actigraphy, diary, and questionnaire for children's sleep patterns. Arch Pediatr Adolesc Med 2008;162: 350-358.

-41 Wolfson AR, Carskadon MA, Acebo C, Seifer R, Fallone G, Labyak SE, Martin JL: Evidence for the validity of a sleep habits survey for adolescents. Sleep 2003;26:213-216.

-42 Pruessner JC, Wolf OT, Hellhammer H, Buske-Kirschbaum A, von Auer K, Jobst S, Kaspers F, Kirschbaum C: Free cortisol levels after awakening: a reliable biological marker for the assessment of adrenocortical activity. Life Sci 1997;61:2539-2549.
3 Dressendoerfer RA, Kirschbaum C, Rohde W, Stahl F, Strasburger CJ: Synthesis of a cortisol-biotin conjugate and evaluation as a tracer in an immunoassay for salivary cortisol measurement. J Ster Biochem Mol Biol 1992;43:683-692.

44 Forsythe AI, Keenan TA, Organick EI, Stenberg W: Computer Science: A First Course. New York, Wiley, 1969.

45 Hatzinger M, Brand S, Perren S, von Wyl A, von Klitzing K, Holsboer-Trachsler E: Hypothalamic-pituitary-adrenocortical (HPA) activity in kindergarten children: importance of gender and associations with behavioral/emotional difficulties. J Psychiatr Res 2007;41:861-870.
46 Heuser I, Yassouridis A, Holsboer F: The combined dexamethasone/CRH test: a refined laboratory test for psychiatric disorders. J Psychiatr Res 1994;28:341-356.

47 Cohen J: Statistical Power Analysis for the Behavioural Sciences, ed 2. Hillsdale, Erlbaum, 1988.

48 Cohen J: The earth is round $(\mathrm{p}<0.05)$. Am Psychol 1994;49:997-1003.

49 Higgins ES, George MS: The Neuroscience of Clinical Psychiatry. Philadelphia, Kluwer, 2007. 